\title{
Research on the Digitalized Communication Planning of Chinese Intangible Cultural Heritage: Taking Ceramics as an Example
}

\author{
Dong Wang ${ }^{1, a}$, Chunhui Zheng ${ }^{2, b *}$ \\ ${ }^{1}$ Harbin Institute of Technology, 92 West Dazhi Street, Harbin 150001, China \\ ${ }^{2}$ Harbin Institute of Technology, 92 West Dazhi Street, Harbin 150001, China \\ âwd_tu_hit@163.com , bchunhuizheng@hit.edu.cn
}

Keywords: Intangible heritages; Ceramics; Digitalization; Manual porcelain making craft of Jingdezhen; Multi-model interaction

\begin{abstract}
In this information era, people are more zealous about the communication of internet cultures, while some intangible heritages which are handed down from one generation to another via words are gradually embarking on the verge of disappearance, leaving the existence of intangible heritages seriously impacted. Recently, the rapid development of digital media technology has provided new opportunities and development space for the preservation and communication of intangible cultural heritages. Based on the research of digitalized design of Chinese intangible heritages, this paper, taking the manual porcelain making crafts of Jingdezhen as an example, produces the artistic effects of simulated ceramics via three-dimensional modelling with new media technologies such as computer animation. On that basis, the computer rendering reinforces the display of the artistic charms of the ceramic textures so that it can compose more ornamental artistic works and improve the public's understanding of traditional ceramics, provides new ideas for the digitalized communication of Chinese intangible cultural heritages.
\end{abstract}

\section{Introduction}

With the approaching of information era as well as the intensification of globalization and modernization, the existence of the traditional Chinese intangible cultural heritages, which are diversified and invaluable, are at stake. Intangible cultural heritages, such as oral traditions, traditional performing arts, fork rituals and traditional craftsmanship, are gradually losing their existential spaces[1]. At the same time, since the cultural ecology has gone through dramatic changes, intangible cultural heritages are impacted at a larger scale than history has ever witnessed. Many cultural heritages which are handed down via words or actions are disappearing, with many traditional craftsmanship on the brink of distinction and large quantities of precious items and documents which enjoy both historical and cultural values are being discarded or lost abroad. Phenomena like misusing or over-exploiting intangible cultural heritages are common. It is high time protecting the intangible cultural heritages in China. The application of current information technologies in the preservation and promotion of intangible cultural heritages lies in the crucial position in the protection of intangible cultural heritages.

\section{Brief Introduction to the History and Development of Ceramics}

History of Ceramics. Ceramics is a general cover name for pottery and chinaware, whose invention is a significant mark in the Neolithic Age and enjoys a long history in China[2]. The English work "China" is an imitation of the pronunciation of Changnan, the ancient Chinese capital of ceramics, which clearly indicate that China is the hometown of ceramics[3]. As much as more than 1,000 years before the Europeans mastered the skills of making chinaware, the Chinese had produced refined ceramics and China was one of the earliest countries to employ ceramics. Due to its excellence in practicability and artistry, the Chinese ceramics have enjoyed high renown in the world. 
The development of traditional ceramics experienced a long time span, which went through the ancient painted pottery to proto-porcelain and onto the development and prosperity of chinaware, corresponding to the process of originating and developing of the Chinese culture.

Ceramics have played significant roles in primitive witchcrafts, totem worshiping, as well as being buried with the dead, sacrificing and religious activities of the civilized ages(refer with: Fig. 1 ). As the development of times and the replacement of different dynasties, ceramics have constantly changing their original appearances and display distinctive artistic features and ornamental devices in each period, of which four categories, namely Guan, Ding, Ge, Jun, $R u$, have attained much fame in the history. In the whole development history of ceramics, each of them have played leading roles for several hundred years. However, during to various natural or anthropic factors, these famous ceramics have also fallen. Even since the Song Dynasty, the manual porcelain making of Jingdezhen remains the only one that retains its fame.

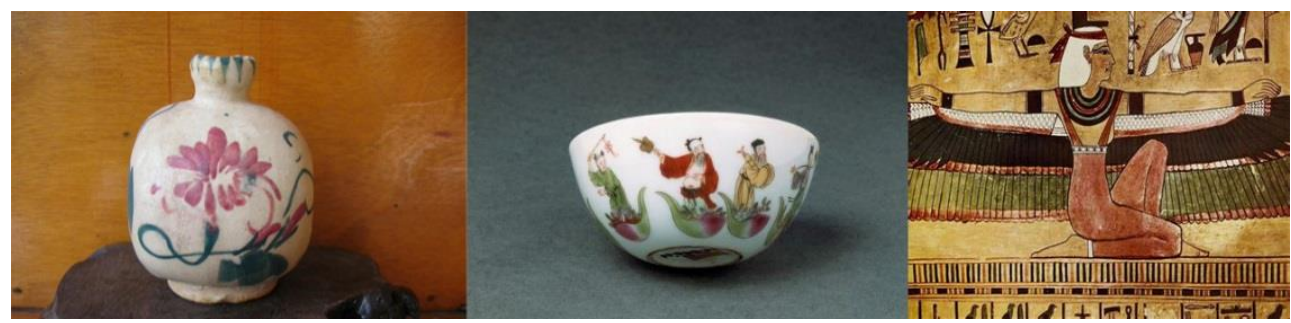

Figure. 1

Current development of ceramics. Chinese cultures are gradually gaining more recognition and affirmation in the world. With the advancement of science and technology, the tide of technology has brought about the dramatic changes in the whole social politics, economy as well as culture[4]. In the tide of technology, traditional handicrafts almost disappeared and the composition of manual handicrafts was one discarded. Since the industrial revolutions, due to the loss of skilled craftsmen, the whole handicraft skills have been replaced by industrial production. Given that the acquisition of handicrafts requires large amount of time inputted in both learning and practice by both the student and the teacher, there are chances that the products and procedures of such handicrafts may be discarded[5], which will certainly be of great loss for the cultural heritages. Not only because that such handicraft articles are significant means in expressing artistry, but also that they represent a very important facet of our cultural heritages and humane civilization.

Currently, the protection of intangible cultural heritages is just on the starting phase and has not been matured. Taking Jingdezhen, the "capital of chinaware", as an example, it has been a historically and culturally renowned city for ceramics for 1,800 years and up till the 1990s, ceramics had remained the pillar industry of this city. (refer with: Fig. 2) However, as time passes, after going through the splendid eras of the greenish white porcelain of Tang Dynasty and Song Dynasty, the blue and white porcelain of the Yuan Dynasty, the rice-pattern decorated porcelain of Ming Dynasty, wucai porcelain of the Qing Dynasty as well as the founding chinaware after the foundation of the People's Republic of China, the ceramics industry have been greatly influenced in its material space due to the impact of market economy and the acceleration of urbanization. In the urbanization of Jingdezhen, activities such as restoring the former city, the constructing of roadways as well as building large construction projects, have resulted in different degrees of damage or even disappearance of many cultural relics, civilian kiln workshops and traces of kilns. Even though a series of salvaging protection measures have been taken, such as building museums of kiln relics in the force places, the previous historical and cultural environment can never be restored. Some scholars have visited the remaining workshops of the "ten ceramics workshops" in 2013 and found that except for a few that have been shifted into cultural and creative industry parks, most of them are in the status of desertion and have been seriously damaged. Even if the local government is proactively protecting the remaining cultural heritages, due 
to the limit of expenses, most of the protection are carried in the protection of frameworks, while the restoring of them are beyond them[6].

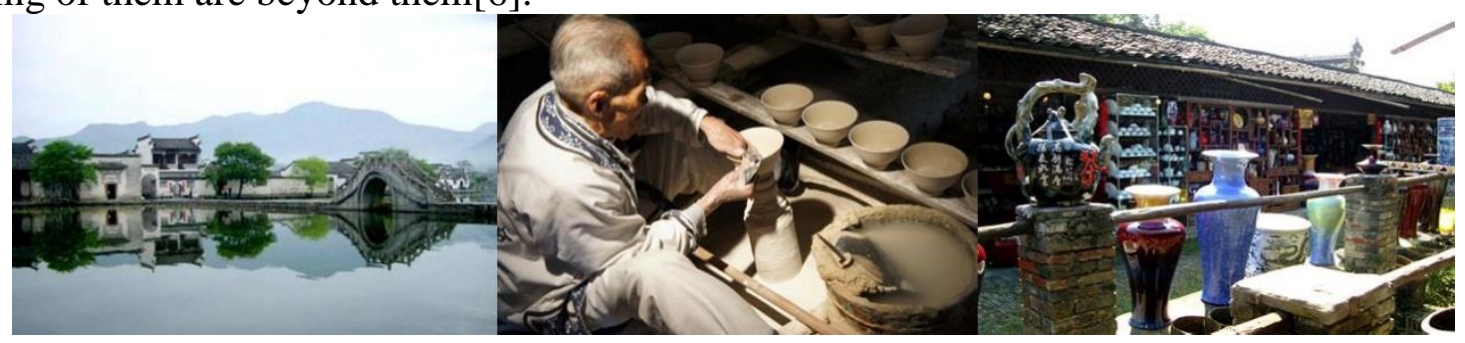

Figure. 2

At this moment, agencies realizing the value of intangible cultural heritages are seeking ways of communication of the intangible cultures in this modern society. It is a good way to return to the previous context, and the virtual reality technology has proved its effectiveness in the reappearance of the former composing environment.

\section{Multimodal Interactive Experience and Communication of Ceramics Virtual Interactive Space}

Based on the above analysis, the roles of virtual ceramics lie not only in the display of the material space and ecological space of space composing of traditional ceramics, but also in rendering the audience to experience the cultural space and its cultural connotations of ceramic handicrafts. Given the development of digitalized technology, which brings new blood in the preservation and communication of intangible cultural heritages, this paper, taking the current research of both home and abroad into consideration, lays the focus in areas such as the development of interactive games, reproduction of virtual reality, the construction of game platforms. Extracting its innovations and employing the elements of traditional ceramics into digital games, or more specifically, the ceramics experience games, and inserting this experience game into the network platform, the technology grants users to take part in the functional interface, network, as well as the forum of the interactive experience, which compose the network carrier of this experience game.

Interactive Design Experience in the Virtual Production of Ceramics.From the perspective of psychology, the information paths through which human beings receive stimulates and making corresponding responses are called channels. The sensing channel and animation channel are responsible for the input and output of information respectively. There are mainly senses of seeing, hearing, touching and acting, while the animation channel consists of various feelings such as hands, feet, bodies, language and expressions, which in interaction with the animation channel, constitutes an interactive means which we call natural interaction[7]. The platform design of the virtual ceramics production is mainly composed of data interaction, image interaction, speech interaction and behavior interactions. From the viewpoint of ergonomics, the whole interactive design influences the feelings of users toward the game experience. In conformity with principles of easy accessibility and adaptability, the game only retains necessary operations while leaving large numbers of operational programs in the back end. In the operational process of each scenario, there are video hints to ensure the experiencing audience have complete information about the experiencing functions of the game, realizing the bilateral interaction of the input and output of information(refer with: Fig. 3)[8]. 


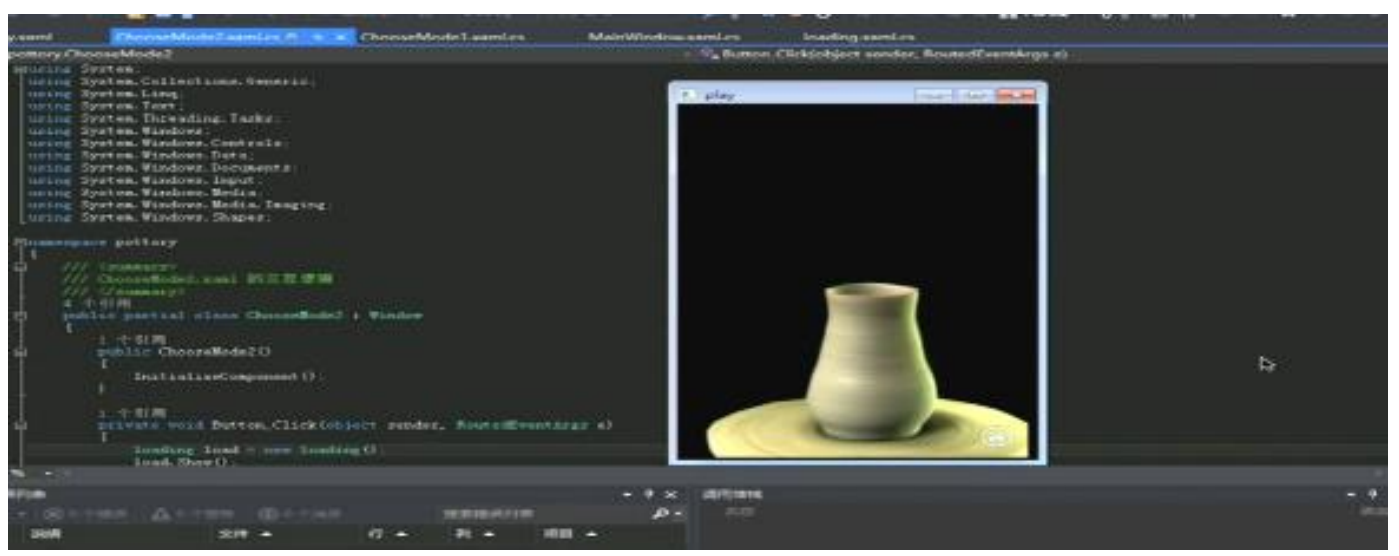

Figure. 3

Sensory Experience in the Production of Virtual Ceramics. The reason why the design chose mobile terminals is that compared with traditional computers, mobile smart terminals, to a larger extent, get rid of the refrain of time and space and due to its smallness and lightness, more portable and easily accessible. The sensing process is accomplished through the sensing channel and the central nervous system. In order to activate the emotions of experiencers, the design employs the multi-composition of auditory perception, visual perception and sensing of touching and adds a feedback design so as to diversify the feedbacks of the game. As a touch-effect themed experience game, the significance of touching towards the virtual ceramics composition platform is without question. Compared with the clicking of mice, due to the thickness of fingers, there exist some precision problems in the finger operation in mobile terminals, which requires that the minimalized size of operational touching controls has to be larger than the square produced by the contact of fingers and the screen[9]. At the same time, given that in the operations of zooming in and zooming out, body activities will be involved, which improves the fun of the game and brings experiencers more real feelings. In the game, each scenario is equipped with corresponding music to simulate the effects of actual operational procedures and provide experiencers with an easy composition environment, which enhances the immersion of the game.

Emotional Experience in the Virtual Ceramics Production. Experience is a subjective component of emotions. Designers usually choose to model the product experience so as to touch the emotional feelings of the users and influence their general sensing of the product[10]. In the level of instinct, the purpose of design is to model people's "first impressions". In the process, styles, color and confrontations of the designing are employed to build appropriate sensory stimulation, of which emotions play a crucial role. As for the design in the level of behaviors, it is mainly about the design of the feelings of experience towards the design, such as excitement, sadness, fidgety and happiness which are produced in the process of experiencing.

\section{Summary}

Ceramics are the Chinese cultural conventions. For computational ceramics, it should not only mimic in effects the traditional handicraft, but also to develop the characteristic artistic effects that are unique to computational ceramics. On the other hand, more diversified ways of communication should be applied to meet the medium demand of different recipients so that the mass could have more convenient and easy access to the information of intangible cultural heritages. Through multi-media presentations and interactive operations, the public may get deeper understandings toward traditional ceramics so that more of them can be attracted to join in the employment of ceramics. This research which takes ceramics as an example, provides new ideas for the digitalized communication of Chinese intangible cultural heritages. 


\section{Acknowledgements}

This paper is a phased research result in the Nation Social Science Foundation's Youth Project-Study on the Innovation Protection Model and Media Communication Strategy of Intangible Cultural Heritage(project number:13CXW016), Heilongjiang Philosophy and Social Sciences Research Planning Project for the Youth-Study on the Digital Contents Communication and Design Model of the "Intangible Cultural Heritage in Heilongjiang Province (project number: 11C038), and Heilongjiang art planning project for the Youth-Study on the Digital Design and Practical Application of Intangible Cultural Heritage in Heilongjiang Province(project number: 11C014)

\section{References}

[1] Convention for the Safeguarding of the Intangible Cultural Heritage. Official journal of standing committee of national people's congress, People's Republic of China. 2006(2): 138-145.

[2] Xiaoqing Chu. Origin and Development of the Space Theory in Ceramics of the Song Dynasty. Hundred Schools in Art. 2008 (06) : 106-108.

[3] Yanzu Li. Internalization and Exchange: From East Asia to the World-Modern Pottery Art in China and Pottery Trend in East Asia. Art \& Decoration. 2005(12)

[4] Lin Meng. On the intangible cultural spaces of traditional handicrafts. Art of Design. 2012 (05) : 76-78

[5] Wenzhi Zhang. Perspectives on the education of pottery in American universities and colleges. Art Journal. 2005(04)

[6] Shuangning Li. Developing Modes of Creative Ceramic Cultural Industry in Jingdezhen. Ceramics college of Jingdezhen. 2012.

[7] Jiemin Gong, Xianqing Wang. The progress and trends of human-computer interaction. Journal of Xidian University. 1998(06)

[8] Yundong. The future of computational human-computer interactive technologies. Digital Space. 2003(02)

[9] Yanjiang Wang, Baozong Yuan. Pen-Based Gesture Recognition in Multimodal Human-Computer Interaction. Journal of Northern Jiaotong University. 2001(02)

[10] Junfeng Chen. Research and findings of cross platform mobile online games based on Unity 3D. Zhongshan University. 2013 